\title{
Some Implications of Aircraft Interference Patterns in Troposcatter Reception
}

\author{
John A. Bradshaw \\ Contribution from General Electric Research Laboratory, Schenectady, New York
}

(Received December 17, 1962; revised January 14, 1963)

\begin{abstract}
Aircraft interference patterns in troposcatter signal records betray the plane's velocity across the link axis. The patterns also reveal the phase variations in the normal signal path. Similarly, the spectra of records free of aircraft patterns reveal the cross-axis velocity of winds aloft. The amplitude distributions of such records often come close to the Rayleigh model but do not follow weather parameters closely. The distributions of ratios and products of correlated amplitudes also fit the Rayleigh model in records free of aircraft reflections.
\end{abstract}

\section{Introduction}

Interference between the normal signal and reflections from aircraft flying in the common volume seen by transmitter and receiver leads to characteristic aircraft patterns in records of the signal level received over troposcatter links. These reflections sometimes disturb TV reception, too. The interference patterns occur frequently in records made of continuous wave transmission at $10 \mathrm{kw}$ and $915 \mathrm{Mc} / \mathrm{s}$ over a 134-mile path from Bedford Airport near Boston to Schenectady, New York. ${ }^{1}$ Usually the patterns seem a nuisance, but they may be worth a second look. They carry information on the aircraft's velocity across the link axis. Moreover, they represent an extreme case of a single predominant scatterer in the midst of scattering from many weaker sources, so they help to clarify features of the spectrum and the distribution of the signal amplitude.

\section{Aircraft Patterns}

To define our notation, we refer to a sideview of the link in figure 1 . The aircraft at $P$, a distance $T$ from the transmitter and $R$ from the receiver, moves along a horizontal line $p$ with velocity $v$ and at a height $h$ above the link axis. If $p$ is projected down on to the horizontal plane through the link axis it will be found to cross it at a distance $x$ from the axis midpoint, and at an angle $\psi$. The total signal path $L$ by way of the aircraft is the sum $T+R$, and for its rate of change we find:

$$
\frac{d L}{d t}=\frac{4 v p \sin ^{2} \psi}{l}\left(1-\frac{4 x^{2}}{l^{2}}\right)^{-1}, v=\frac{d p}{d t},
$$

\footnotetext{
1 Under Contract AF 19(604)-1723 with the AF CRC.
}

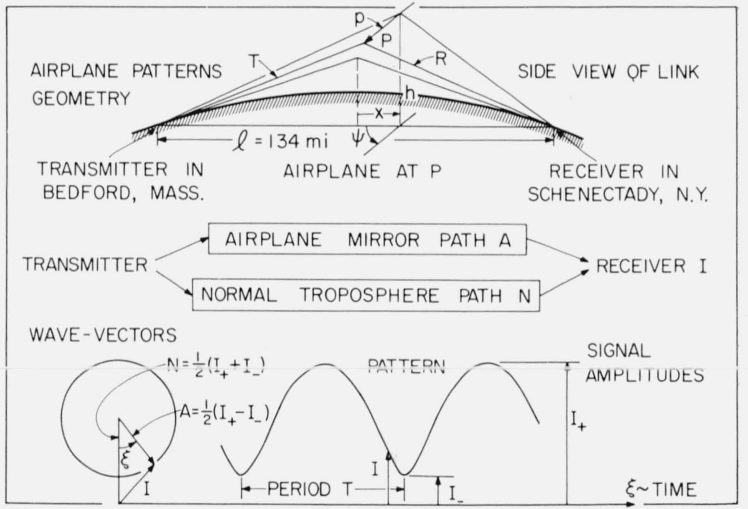

FIGURE 1. Notation for airplane patterns.

under the assumption that $h$ and $p \sin \psi$ are much less than $l / 2$. The second time derivative of $L$ is

$$
\frac{d^{2} L}{d t^{2}}=\frac{4 v^{2} \sin ^{2} \psi}{l}\left(1-\frac{4 x^{2}}{l^{2}}\right)^{-1}
$$

Similar formulas are given by Pokorny in an article in Czechoslovakian not readily available [Pokorny, 1961]. If the aircraft contributes a wave $A$ and the normal path contributes a wave $N$ to the receiving antenna, as sketched in the lower part of figure 1 , the receiver output $I$ represents the vector sum of $N$ and $A$. We take $N$ as the phase reference, and we use $\xi$ to indicate the phase difference between $N$ and $A$. As $\xi$ changes, $I$ traces the pattern characteristic of aircraft interference. If, for example, $|A|$ is less than $|N|$, as in figure 1 , the pattern gives us the original wave amplitudes $|N|=1 / 2\left(I_{+}+I_{-}\right)$ and $|A|=1 / 2\left(I_{+}-I_{-}\right)$, where $I_{+}, I_{-}$are the extremes of $I$. 

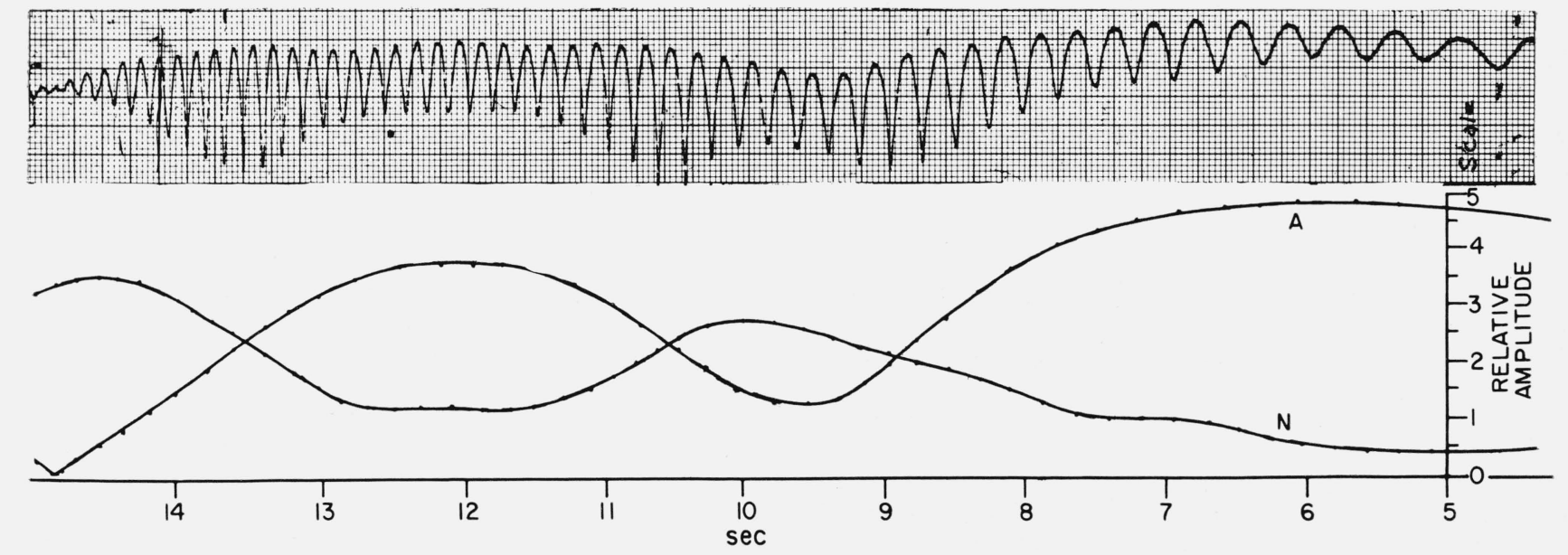

Figure 2. A typical airplane pattern and its analysis.

In figure 2, one analysis of a sample $10 \mathrm{sec}$ of interference has yielded the variations of $|A|$ and $|N|$ plotted beneath the record. In figure 3 , the first graph includes these 10 sec, with $A^{2}$ plotted against time on a log-log scale; the second graph comes from a similar record. Time in each case is measured from the apparent center of the pattern. Both curves have knees - at about 14 and 6 sec respectively - and they fall off beyond the knees at slopes $m$ of 6 and 10 respectively (ignoring the fine structure in the first pattern). Thus they can be approximated by the formula $P \sim\left(1+\left[t / t_{0}\right]^{2}\right)^{-m / 2}$.

Now the Booker and Gordon [1950] formula for scattering from transparent inhomogeneities indicates that the power scattered will be proportional

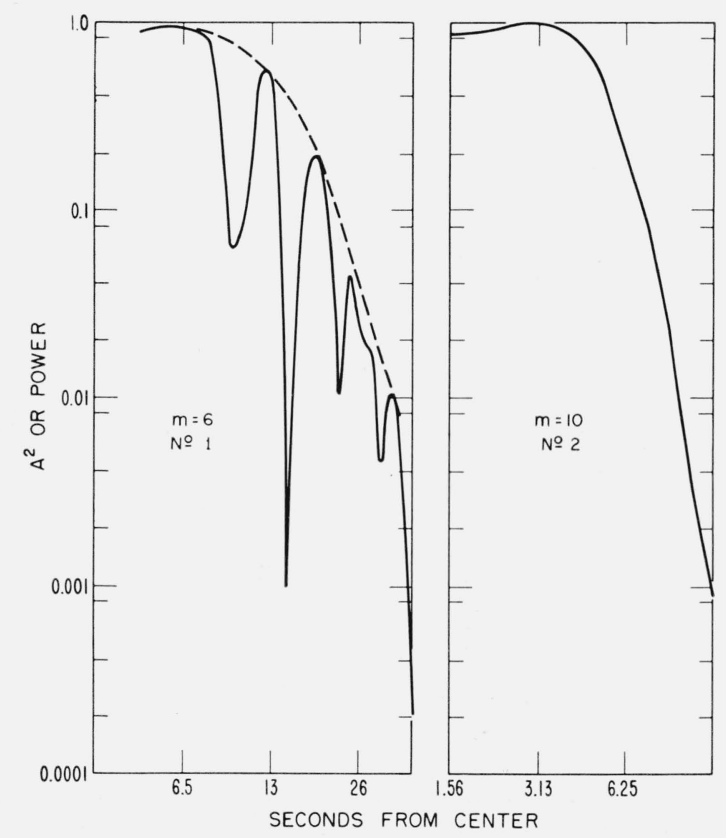

Figure 3. Power in the signal scattered by an airplane, No. 1 and No. 2. to $\sin ^{-m} \theta / 2$, where $\underline{\theta}$ is the angle at $P$ in figure 1 , between $T$ extended and $R$, and $m$ is about 4 . For angles in the range of interest we have

$$
P \sim \sin ^{-m} \theta / 2 \sim\left(1+[z / h]^{2}\right)^{-m / 2} .
$$

Here $h$ is the height of the scatterer, and $z=p \sin \psi$ is its horizontal distance from the link axis. If (3) is plotted on $\log -\log$ scales for various values of $m$, we obtain figure 4 , which consists of curves resembling those in figure 3 .

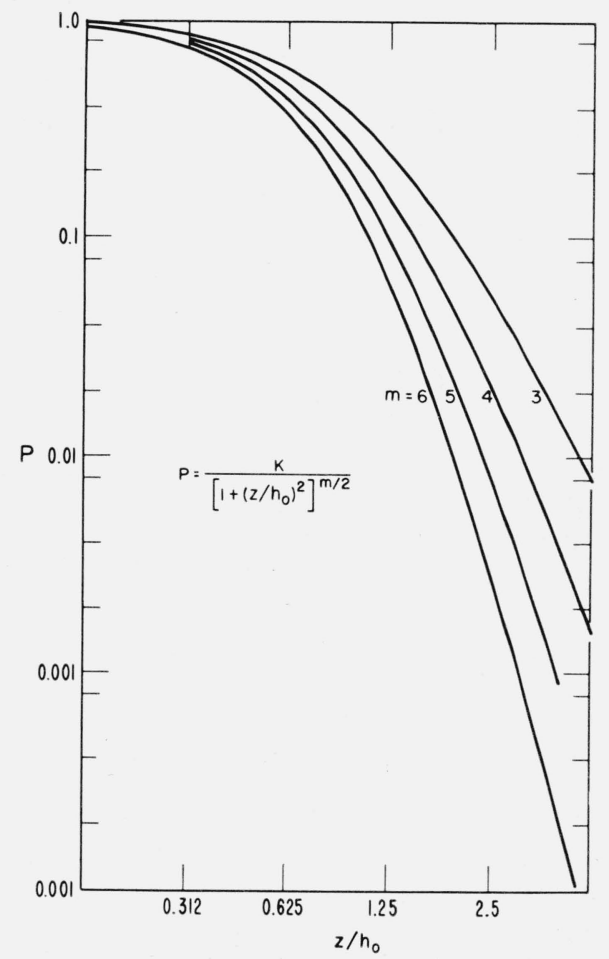

FIguRE 4. A model of power scattered against off-axis position. 
Reflections from shiny and opaque objects, however, clearly will not depend on $\underline{\theta}$ in the simple manner of $P$ in (3). The plane's velocity across the axis, $v$ $\sin \psi$, may be found from patterns like that in figure 2 , and then $z_{k}$ at the knee of the corresponding power curve may be obtained. We find 4,700 and 3,500 ft off axis, respectively, for $z_{k}$ in the patterns analyzed in figure 3 . These values of $z_{k}$ would equal the plane's height above the link axis, if (3) applied to scatter from aircraft; but the plane must, of course, be above the bottom of the common volume at 4,500 $\mathrm{ft}$ (calculated from an earth with four-thirds the true radius). Again, these two values of $z_{k}$ fall very much short of indicating the half-width of the common volume: the receiver with an 18-ft dish and the transmitter with a 28 -ft dish "see" regions 40,000 and $26,000 \mathrm{ft}$ in half-width respectively at midlink. Further data will be required to interpret correctly the shapes of the power curves in figure 3 .

We now obtain the velocity of the aircraft across the axis. Returning to figures 1 and 2 , we note that the period $T$ between adjacent minima of a pattern of interference can be fixed fairly accurately. If $M$ is the length of the normal signal path, the difference $(L-M)$ will change by a signal wavelength, $\lambda=0.328$ $\mathrm{m}$, between adjacent minima:

$$
\frac{d(L-M)}{d t}=\frac{\lambda}{T} .
$$

Except when the plane is directly above the link axis, or moving parallel to the axis, the chief term in (4) will be $d L / d t$ rather than $d M / d t$, since the plane's velocity much exceeds that of the winds aloft. If we plot $\lambda / T$ from a pattern such as appears in figure 2 , we obtain in fact a straight line perturbed randomly by the small effects of $d M / d t$. As shown in (2), the slope of this line represents the square of the cross-axis velocity of the plane. In figure $5, \lambda / T$ from four records is smoothed out and plotted against the time measured from the pattern center. Typical aircraft velocities are obtained.

Having identified the component $d L / d t$ in plots of $\lambda / T$, we turn next to $d M / d t$, and consider the phase stability of the normal path. It is difficult to monitor the phase of a tropospheric signal; neither reference to very stable oscillators at both path terminals, nor the retransmission of the signal back to the transmitting site seemed feasible for us [Angell, Foot, Lucas, and Thompson, 1958]. We did, however, study the frequency dependence of the phase (or of the path length $M$ ) through data obtained by modulating the $915 \mathrm{Mc} / \mathrm{s}$ signal at $1 \mathrm{Mc} / \mathrm{s}$. This gave 914 and $916 \mathrm{Mc} / \mathrm{s}$ sidebands, hereafter labeled $a$ and $c$, while $b$ refers to the carrier frequency. The $\lambda / T$ analysis can be made of each component in records of aircraft interference; figure 6 gives an example. For the 14 sec analyzed from records of the simultaneous amplitudes of the $a, b$, and $c$ signals (separated by filters), we find apparently unrelated variations of $\partial M(f, t) / \partial t$ around a line of constant slope representing $d^{2} L / d t^{2}$.

Continuing the analysis of this record, figure 7 gives in the dashed lines in the upper graph the

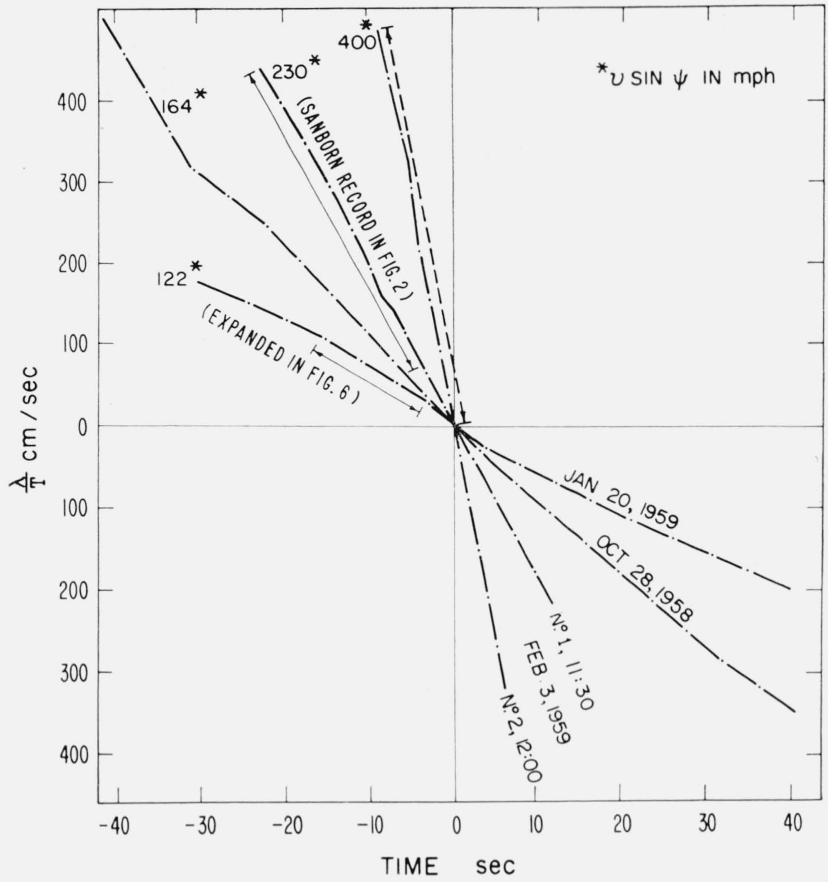

Figure 5. Analysis of four representative aircraft patterns.

differences $\partial\left(M_{i}-M_{b}\right) / \partial t, i=a$ or $c$, between each sideband and the carrier $b$, for 7 of the 14 sec used in figure 6 . Integrals of these curves from an arbitrary point in the - 17th sec give the curves "upper" and "lower" in the lower graph of figure 7. In this record the frequency dependence of $M$ is small, $\pm 2 \mathrm{~cm}$ in 134 miles or two parts in $10^{7}$, for a change in wavelength of one part in $10^{3}$. The "sum" curves in figure 7 represent the second difference $Q(f, t)$ and its integral:

$$
\begin{gathered}
Q=\frac{\lambda}{T_{a}}+\frac{\lambda}{T_{c}}-2 \frac{\lambda}{T_{b}} \mid \\
\int_{t 0}^{t} Q d t==(\Delta f)^{2} \frac{\partial^{2}}{\partial f^{2}}\left\{M(f, t)-M\left(f, t_{0}\right)\right\} .
\end{gathered}
$$

The integral of $Q$ may be recovered at the receiver without a "comparison" signal reflected from aircrait. The circuit used for this operation, designed by Dr. L. G. Abraham, Jr., mixed each sideband with the carrier to obtain two signals at $1 \mathrm{Mc} / \mathrm{s}$, and it compared instants of zero-crossing of these 1 $\mathrm{Mc} / \mathrm{s}$ signals. The output was the second phase difference:

$$
\Phi=\phi_{a}+\phi_{c}-2 \phi_{b}=2 \pi\left(\frac{M}{\lambda_{a}}+\frac{M}{\lambda_{c}}-2 \frac{M}{\lambda_{b}}\right)=\frac{2 \pi}{\lambda} \int Q d t .
$$

The phase $\phi_{a}$ refers, of course, to the lower sideband written in the form $I_{a}(t) \cos \left(\omega_{a} t+\phi_{a}\right)$, and similarly for $\phi_{b}$ and $\phi_{c}$. A typical record of $\Phi(t)$, about a minute long, made on August 12, 1959, appears as 


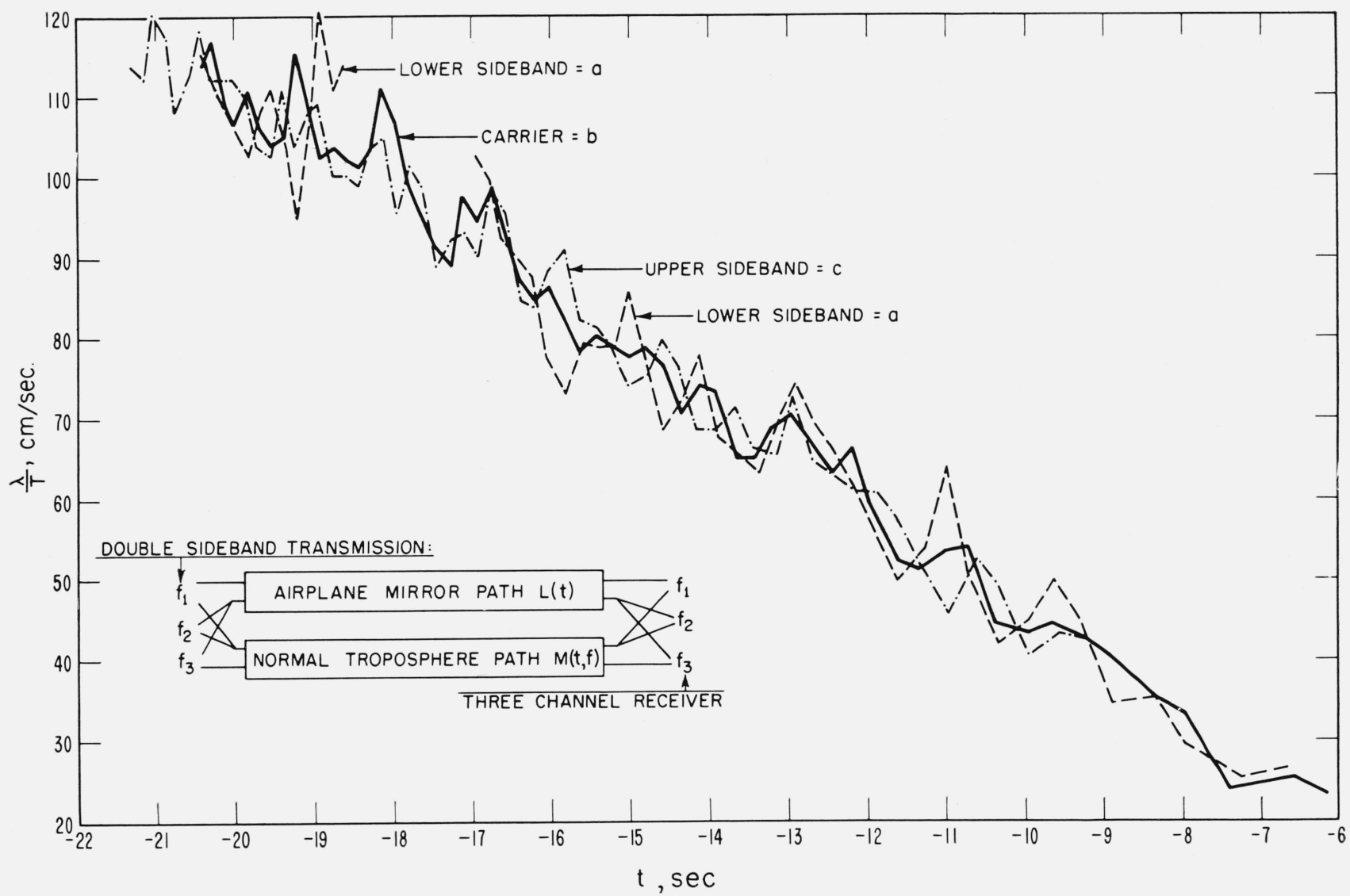

FigurE 6. Variations of $\lambda / \mathrm{T}$ at three frequencies over the same fifteen second interval.

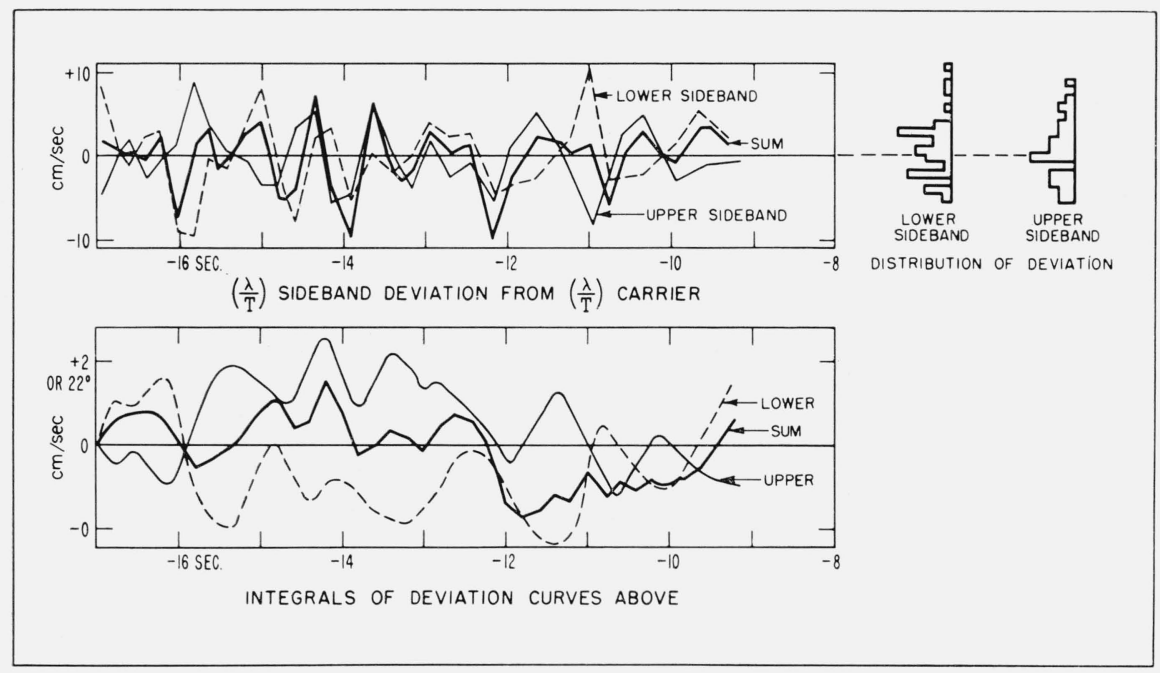

Figure 7. Phase fluctuation differences between sidebands and carrier.

the top trace in figure 8 . The other three traces in this figure give the amplitudes of signals $a, b$, and $c$. The arrows on these traces indicate the points of deep fade; the trace of $\Phi$ shows rapid changes at these instants. The discontinuities in the trace occur wherever $\Phi$ shifts over $180^{\circ}$ either side of the (arbitrary) origin, and represent a periodicity in the instrument.

The variation of $\Phi$ during this relatively calm period approached $720^{\circ}$. Changes of more than 


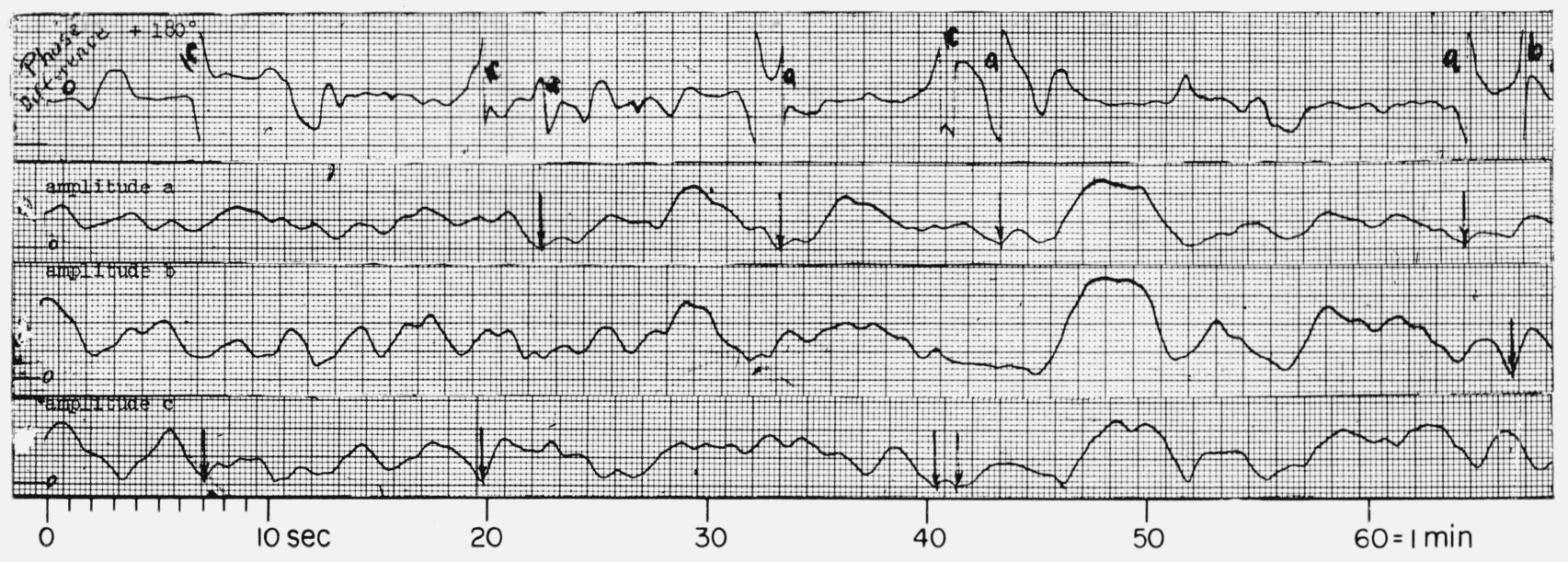

Figure 8. Records of three envelopes, and second difference of phases.

$720^{\circ}$ in a second occur in records of greater disturbance. Nevertheless, these records show that $\partial^{2} M / \partial t^{2}$ changes gradually; sudden and wide fluctuations in $\Phi$ like $180^{\circ}$ in a millisecond would give spikes such as occur only when a signal vector passes near zero in a deep fade.

\section{Spectra}

Much of the analysis and notation used to explain aircraft patterns helps also to describe the spectra of the amplitude records of signals not contaminated by aircraft reflections. The spectra we consider here were obtained by running several minutes of record, mixed with añ aüdio signal, through a narrow filter $(1 \mathrm{c} / \mathrm{s}$ wide, centered at $50 \mathrm{c} / \mathrm{s})$ and integrating the filter output. Some records were processed for us at the University of Texas. These spectra appear to us to have resulted from scattering by a number of centers in fairly uniform motion across the path axis. The centers are presumably "blobs" of uneven dielectric constant in the atmosphere, although their conformation must vary as much as the shapes of visible clouds, and may include rough or rippled layers as well as spheroids. The amplitude of radiation received from a blob will, of course, depend on its size and intensity, but also on the scattering angle, in accordance with (3). Thus for $z / h$ larger than 1 , the blob's contribution, beating against the average of a group of other wave vectors, will fall in amplitude as its $z / h$ increases. The spectra as a whole tend to take the form of figure 4 . Figure 9 shows examples, for a succession of 7 min recorded May 8, 1958, and reproduced here to show the consistent appearance of a knee, and a drop-off with exponent about $m=4$. The 8 th $\mathrm{min}$, contaminated by aircraft, has a different and typical shape, a plateau or bump on the drop-off.

The "blobs" drift with the air movements, and scatter wavelets that differ slightly in frequency from the transmitted frequency $f_{b}$. The Doppler shift depends, of course, on the rate of change of the path-length $M$ via the blob;

$$
\Delta f=f-f_{b}=\frac{f_{b}}{c} \frac{d M}{d t}=\frac{4 v p(\sin \psi)^{2}}{\lambda_{b} l}
$$

assuming a horizontal drift and $(2 x / l)^{2}<<1$. If the blob happens to move so $M$ is constant, that is, on the surface of an ellipse of revolution with foci at the

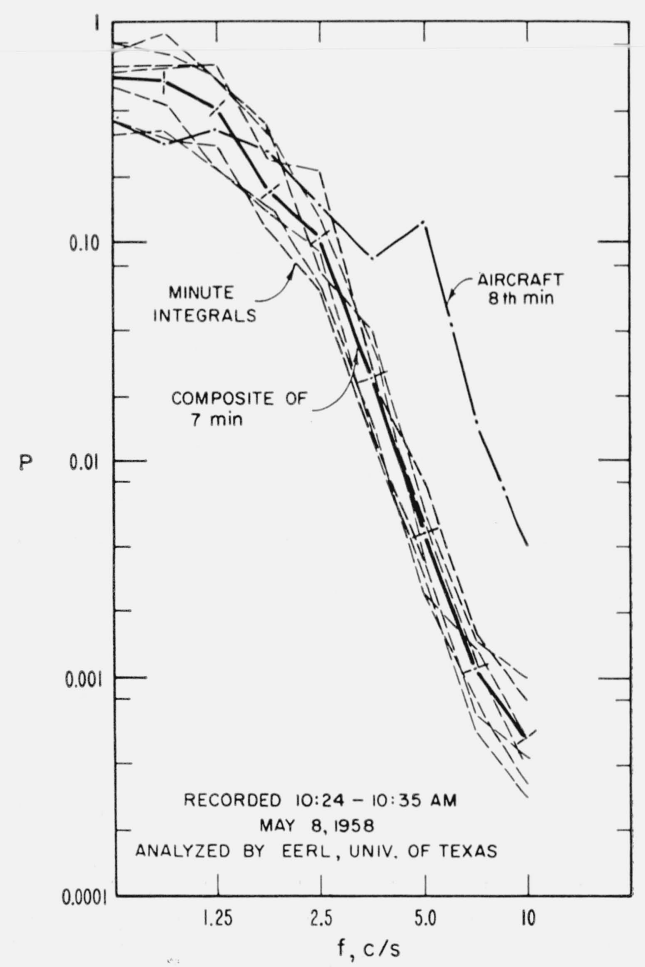

Figure 9. Power density spectra from May 8, 1958. 
two antenna sites at the link ends, then $\Delta f=0$, but when the blob moves perpendicular to such a surface, $\Delta f$ is a maximum for a given speed [Laaspere, 1958]. Usually, and particularly in winter, the winds carry scatterers horizontally, and we may solve for their cross-axis velocity, using for $\Delta f$ the frequency at the knee of the spectrum, our link dimensions for $l \lambda$, and for $h=z$ the estimate of $4,000 \mathrm{~m}$ :

$$
d z / d t=v \sin \psi=4.42 \Delta f \mathrm{~m} / \mathrm{sec} .
$$

Figure 10 shows four spectra from records taken in December 1957, in extremes of weather. From $\Delta f$ at the knee of each curve we obtain cross-axis velocities of the scatterers. In figure 11 we compare these with actual velocities of winds aloft. The latter come from soundings [U.S. Department of Commerce, Weather Bureau, Daily Series, Synoptic Weather Maps, Part II, 1957] at the Albany Weather Station, obtained at 7 a.m. or 7 p.m. Although Albany is on the link axis, it lies 60 miles from the axis midpoint; moreover, our records were made between 8:30 a.m. and 4:30 p.m. so the Albany weather data are not ideal. Fortunately, winds aloft are fairly persistent phenomena, characteristic of large areas. In figure 11, points No. 1 through No. 4 correspond to curves so numbered in figure 10 . The extreme point No. 1 falls nearly on the diagonal line of complete agreement between spectral and weather velocities. Points No. 7 and No. 10 also fall on it, while No. 14 is the worst case. When No. 14 was recorded, March 26, 1958, the winds aloft, although between 30 and $40 \mathrm{~m} / \mathrm{sec}$, were almost parallel to the link axis. A change of $10^{\circ}$ in wind direction would put No. 14 on the diagonal.

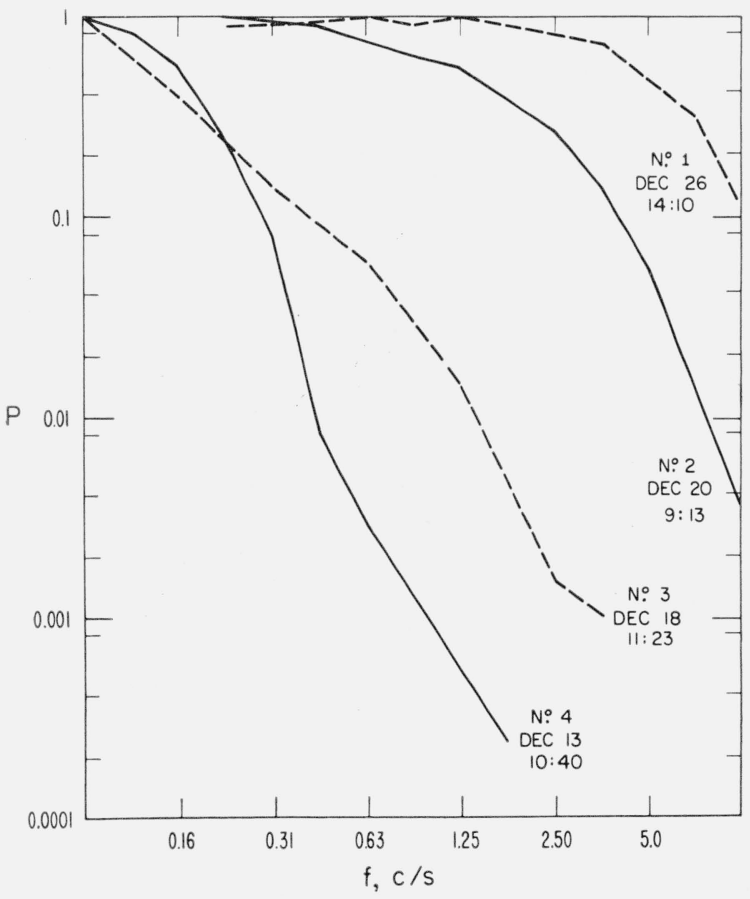

FIgure 10. Spectra from extremes of weather.

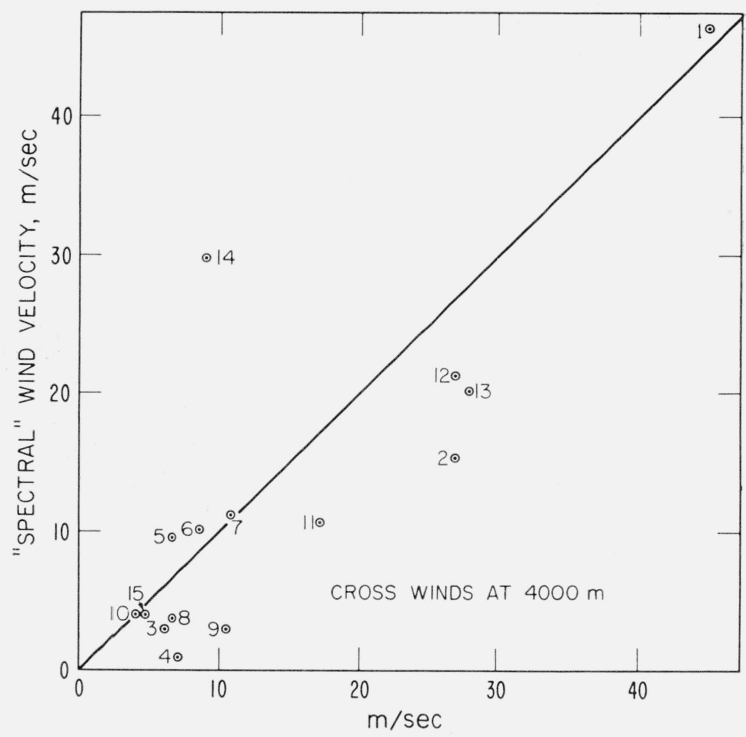

Figure 11. Correlation of cross winds and spectrum.

Although for the aircraft patterns we found $m$ from 6 to 10, most spectra drop off at a slope from 4 to 6 . The slope of the spectra must depend on the distribution of scatterers in height and on the antenna apertures if these are narrow, according to suggested modifications of the Booker-Gordon formula [Villars and Weisskopf, 1955].

\section{Distributions}

The addition of a large number of wave vectors, none of them predominant, arriving with random phases from an assortment of scattering centers, yields a resultant wave vector whose amplitude tends, as a consequence of the central limit theorem [Lawson and Uhlenbeck, 1950], to have the Rayleigh distribution

$P(r) d r=\left(r / a^{2}\right) \exp \left(-r^{2} / 2 a^{2}\right) d r$.

An intensive analysis of $2 \frac{1}{2} \mathrm{hr}$ of our records by $\mathrm{R}$. G. Finney [1958] showed sections, particularly in records from calm days, with long periods of stationary rms levels, and distributions satisfying a $\chi^{2}$ test for a Rayleigh population, at the 5 percent level of probability of rejecting the hypothesis, when the distribution was, in fact, Rayleigh.

We also collected samples of the cumulative distribution, obtained by reading the recorded signal envelope into a set of clocks controlled by relays triggered at preset levels (a Gates multilevel recorder). The results for seven consecutive minutes of clear record and $4 \mathrm{~min}$ (dashed curves) contaminated by aircraft are given in figure 12 . There we plot the cumulative distribution, the fraction $P$ of the record that lies below the amplitude $r$ (abscissa), against $X=$ $r / r_{m}$ (ordinate), where $r_{m}$ is the median signal level. Curve No. 1 coincides with the Rayleigh model, and the succeeding minutes yield curves not far from it, until the aircraft interference begins. 
If we have interference between two equal scatterers, separated a distance $d$, and at a height $h$, drifting at a velocity $v$ across the link axis, the signal reception may take the following form:

$I(t) \sim\left(f^{2}+g^{2}-2 f g \cos u t\right)^{1 / 2} ;$

where

$1 / f=1+(v t / h)^{2}, 1 / g=1+(v t-d)^{2} \cdot h^{-2}$,

and

$u=2 \pi / \lambda \cdot\left(d\left(M_{f}-M_{g}\right) / d t\right)$.

The distribution of $I(t)$ for $d / h=6$, accumulated in the interval $0<v t<d$, is plotted as the dotted curve in figure 12, and may help to explain the curve for the eleventh minute.

Figure 13 shows distributions for the records from December 1957, from which the spectra in figure 10 were taken. Clearly the distributions give no indication of the weather: the 20th and 26th had strong: winds aloft and high frequency knees in their spectra, while the 13th and 18th are calm days, yet the 18th and 20 th have similar distributions and the 13 th and 26th are also paired.

Departures from the Rayleigh distribution can sometimes be represented by a single scatterer predominant among small random scatterers. The lines in figure 14, from a numerical intergration by Norton, Vogler, Mansfield, and Short [1955], show distributions for various values of $k$, the ratio of the rms sum of small scattering vector amplitudes to the predominant vector's amplitude. (The distribution is Gaüssian on the wave vecior plane, but the center is displaced from the origin by the predominant vector.) Data from a record without obvious aircraft patterns, taken April 1958, appear as dashed lines on figure 14 ; the original data were close to Rayleigh above median amplitude, but below Rayleigh for lower amplitudes. Adding $4.3 \mu \mathrm{v}(13 \%$ of the median $33 \mu \mathrm{v}$ ) to each point moved the data to overlap the $k=1$ curve over its whole length. This type of change enabled us to fit many curves to the Norton model. However, in each case the voltage increment is different, and does not, apparently, correct an error in data. We merely note that some distributions depart from modified Rayleigh as if by displacement of the zero level.

In studies of the bandwidth of the tropospheric path, a parameter of interest is the correlation $\rho$ between received amplitudes of signals separated slightly in frequency. This correlation is subject to statistical fluctuations, and depends also on weather conditions in the common volume. One can process the amplitude records to give the average values, $\left\langle r_{1} r_{2}\right\rangle$ or $\left\langle r_{1} / r_{2}\right\rangle$, and with these determine $\rho$ from formulas such as we give in an appendix; alternatively one can find the cumulative distributions of $r_{1} r_{2}$ and $r_{1} / r_{2}$, and determine the correlation by comparison with curves plotted in figure 15. An example of the second method, which provides a consistency check in the shape of the curve, appears in figures 16

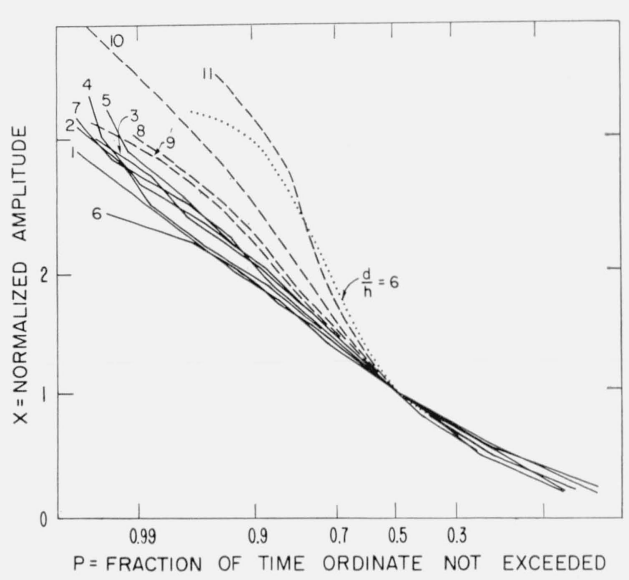

Figure 12. Amplitude distributions for eleven minutes, May 8,1958 .

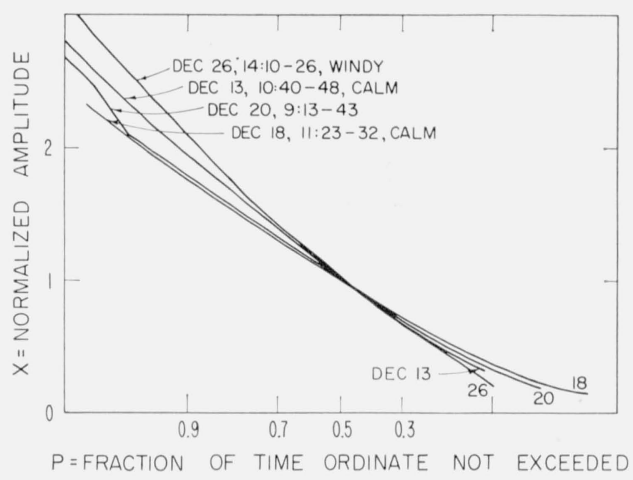

FIgure 13. Amplitude distributions in extremes of weather.

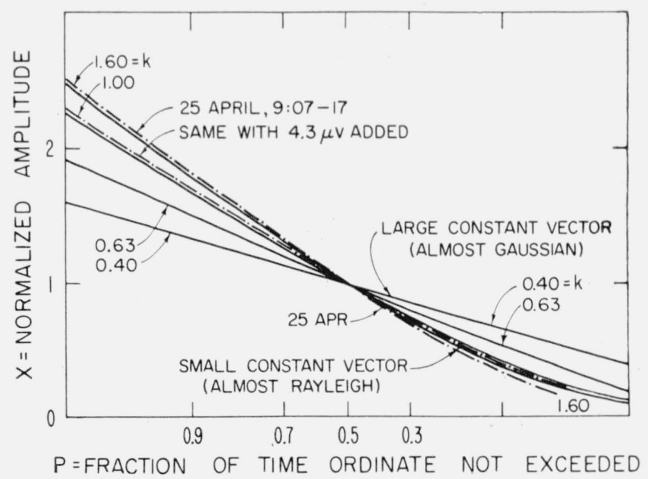

FIgure 14. Distribution of amplitude of sum of a constant vector and random vectors.

and 17. The data analyzed in this way from records made August 5, 1959, are typical of a dozen so processed. They represent here about $4 \mathrm{~min}$ of normal record, and $1 \mathrm{~min}$ of aircraft interference.

The normal ${ }^{2}$ distribution at all three frequencies, $a, b$, and $c$, is close to Rayleigh in figure 16 , and the

2 "Normal" is used here as "commonly occurring," rather than normal in the statistical sense. 


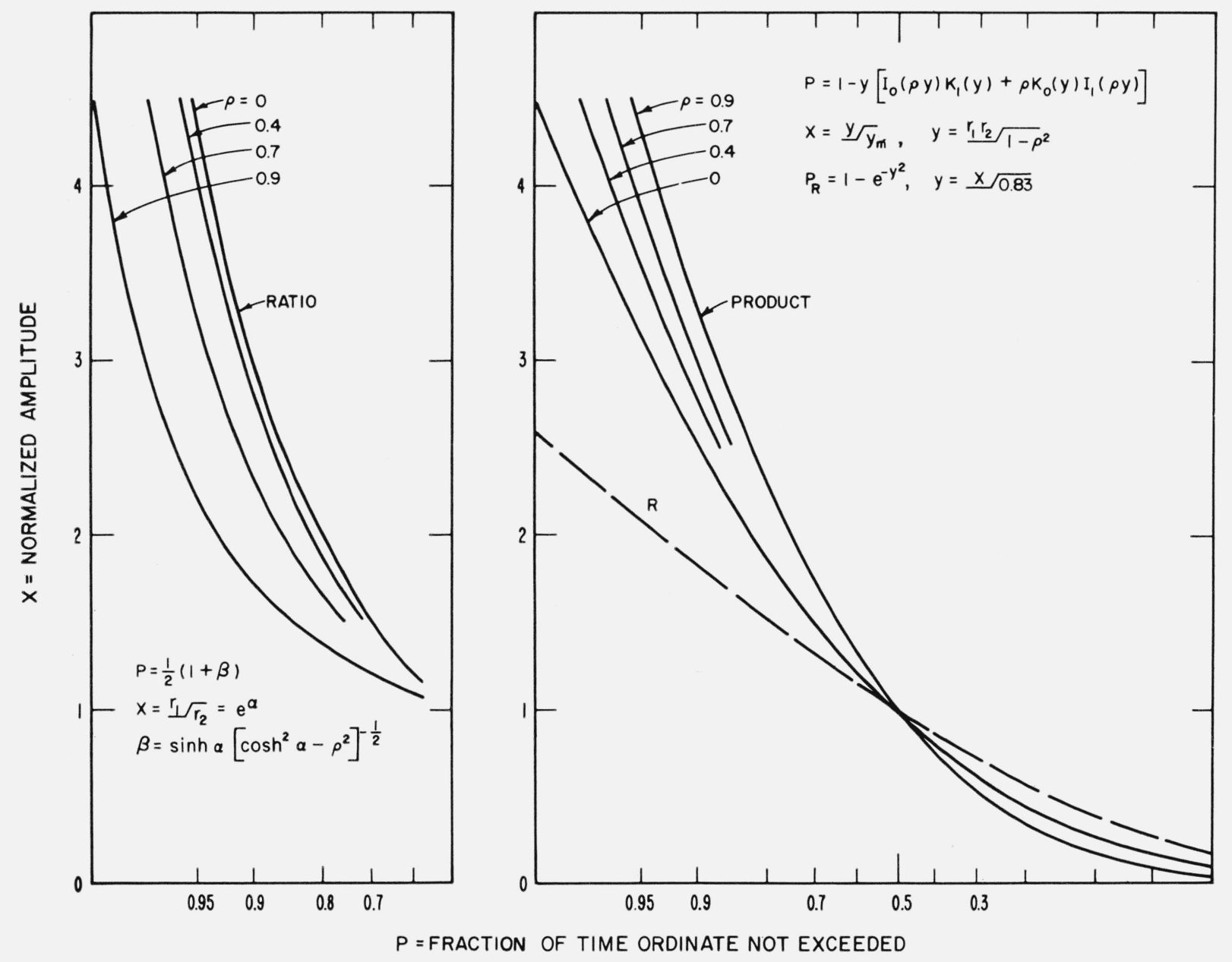

FIGURE 15. Distributions of functions of two correlated Rayleigh amplitudes.

ratios are distributed along curves parallel to the models in figure $15 .^{3}$ In figure 17 , the products from the normal records also follow the model curves, but the products involving aircraft patterns, as might be expected, have more and higher peaks. However, again in figure 17 , the ratios from aircraft patterns are less disturbed than the products. Finally we compare the correlation parameter $R$, determined from $\left\langle r_{1} r_{2}\right\rangle$, with $\rho^{2}$, determined from the cumulative distribution of $r_{1} / r_{2}$, for each pairing of the three normal records:

$\begin{array}{lcc}\text { Pair } & R & \rho^{2} \\ (a, b) & 0.32 & 0.30 \\ (b, c) & 0.49 & 0.50 \\ (c, a) & 0.25 & 0.20\end{array}$

(The definition of $R$, and its relation to $\rho$ are given in the appendix.)

\footnotetext{
3 The ratio distributions are "double": points for $X$ less than 1 are plotted as $1 / X$, and the sign of $\beta$ is changed, so the distributions of $r_{1} / r_{2}$ and $r_{2} / r_{1}$ are not dis$1 / X$, and the sign of $\beta$ is changed, so the distributions of $r_{1} / r_{2}$ and $r_{2} / r_{1}$ are not dis$X>1$ and mirror points $X<1$ fall on the same curve, and are averaged in plotting $X>1$ and mirror
figures 16 and 17 .
}

\section{Summary}

Airplane interference patterns in records of signal amplitude betray the plane's cross-axis velocity, and the phase variations of the normal path. The interference is an exaggerated form of scattering from a predominant scatterer in the presence of random signals from many small ones. It markedly affects both the spectrum and distribution of the envelope record. The spectrum in the absence of aircraft can be related to a model of scatterers drifting with the winds aloft. The correlation of signal amplitudes in nearby frequencies fits a Rayleigh model for the joint distribution, as represented by derived distributions for the product and ratio of signal amplitudes.

The receiving equipment and the phase comparator were designed and assembled under the supervision of Dr. L. G. Abraham, Jr., who also participated in the studies of correlation and effective bandwidth and other parts of the project. J. S. Brookman and T. E. Kotary processed the data. 


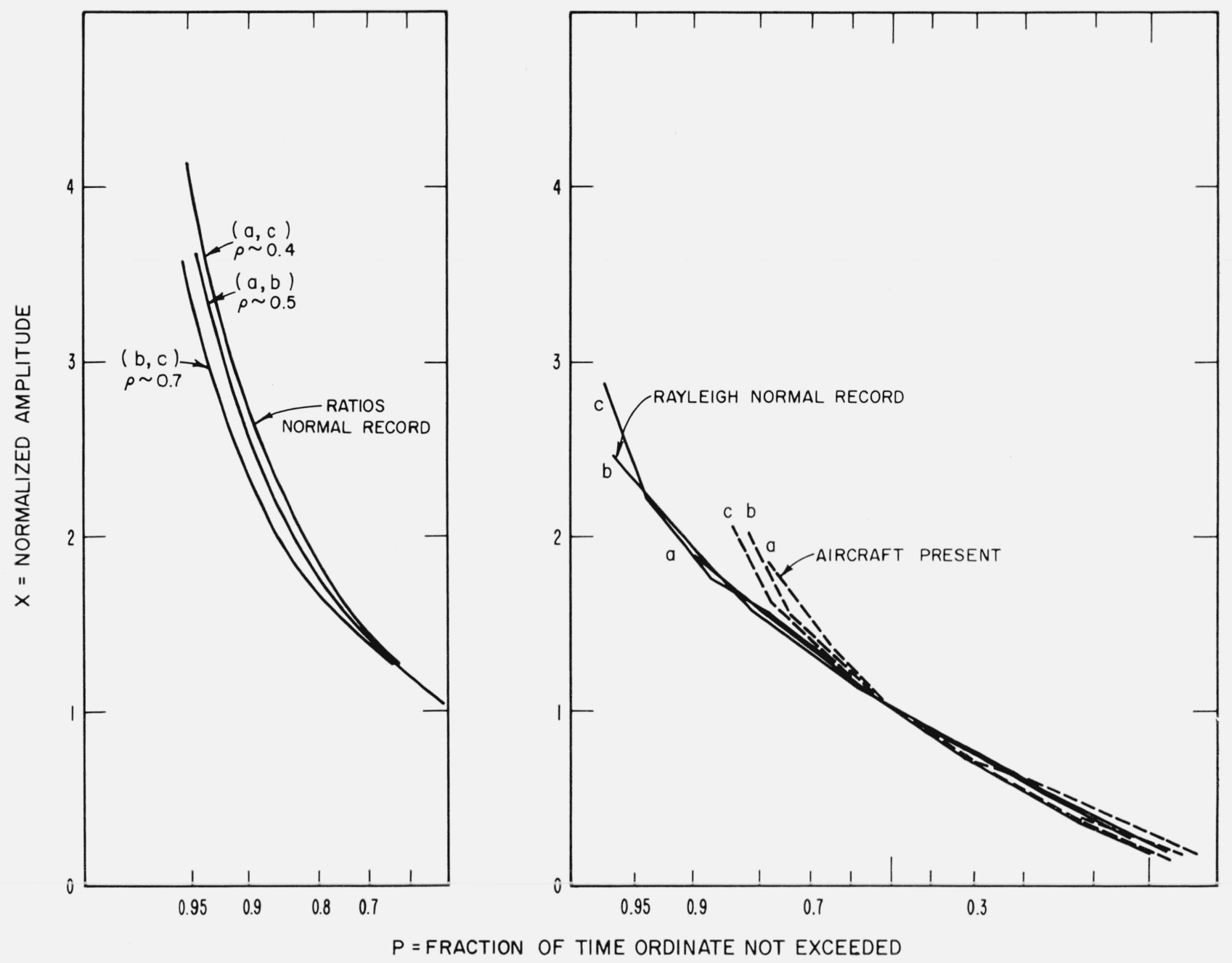

FIGURE 16. Distributions of amplitudes and amplitude ratios at three frequencies, taken August 5, 1959.

\section{References}

Angell, B. C., J. B. L. Foot, W. J. Lucas, and G. T. Thompson (1958), Propagation measurements at $3,480 \mathrm{Mc} / \mathrm{s}$ over a 173 mile path, Proc. Inst. Elec. Engrs. 105, Pt. B. Supp. $8,128-142$.

Booker, H. G., and W. E. Gordon (April 1950), A theory of radio scattering in the troposphere, Proc. IRE 38, 401-412.

Finney, R. G. (June 1958), Short-time statistics of tropospheric radio wave propagation, 58-RL-2030, General Electric Research Laboratory, Schenectady, N.Y.

Finney, R. G. (Jan. 1959), Short-time statistics of tropospheric radio wave propagation, Proc. IRE 47, 84.

Hirai, M., M. Fukushima, and Y. Kurihara (Sept. 1960), Correlation between amplitudes of radio waves of different frequencies in UHF beyond-the-horizon propagation, J. Radio Res. Lab. (Japan) $\boldsymbol{\gamma , 5 0 9 - 5 2 9 .}$

Laaspere, T. (Aug. 1958), An analysis and re-evaluation of the role of horizontal drift in producing fading in tropo- spheric scatter propagation, Res. Rpt. EE380, Cornell University, Ithaca, N.Y.

Lawson, J. L., and G. E. Uhlenbeck (1950), Threshold signals, 61-62 et al. (McGraw-Hill Book Co., Inc., New York, N.Y.).

Norton, K. A., L. E. Vogler, W. V. Mansfield, and P. J. Short (Oct. 1955), The probability distribution of a constant vector plus a Rayleigh-distributed vector, Proc. IRE 43, 1354-1361.

Pokorny, J. (1961), Determination of the velocity and position parameters of the aircraft from interference in the longdistance reception of very short waves (in Czechoslovakian), Slaboproudy Obzor 22, 487-491.

U.S. Department of Commerce (1957), Weather Bureau, daily series, synoptic weather maps, Part II; winds aloft checked data.

Villars, F., and V. F. Weisskopf (Oct. 1955), Scattering of radio waves by turbulent fluctuations of the atmosphere, Proc. IRE 43, 1232-1239.

Wheelon, A. D. (1959), Radio-wave scattering by tropospheric irregularities, J. Research NBS (Radio Prop.) 63D, No. 2, $205-233$. 


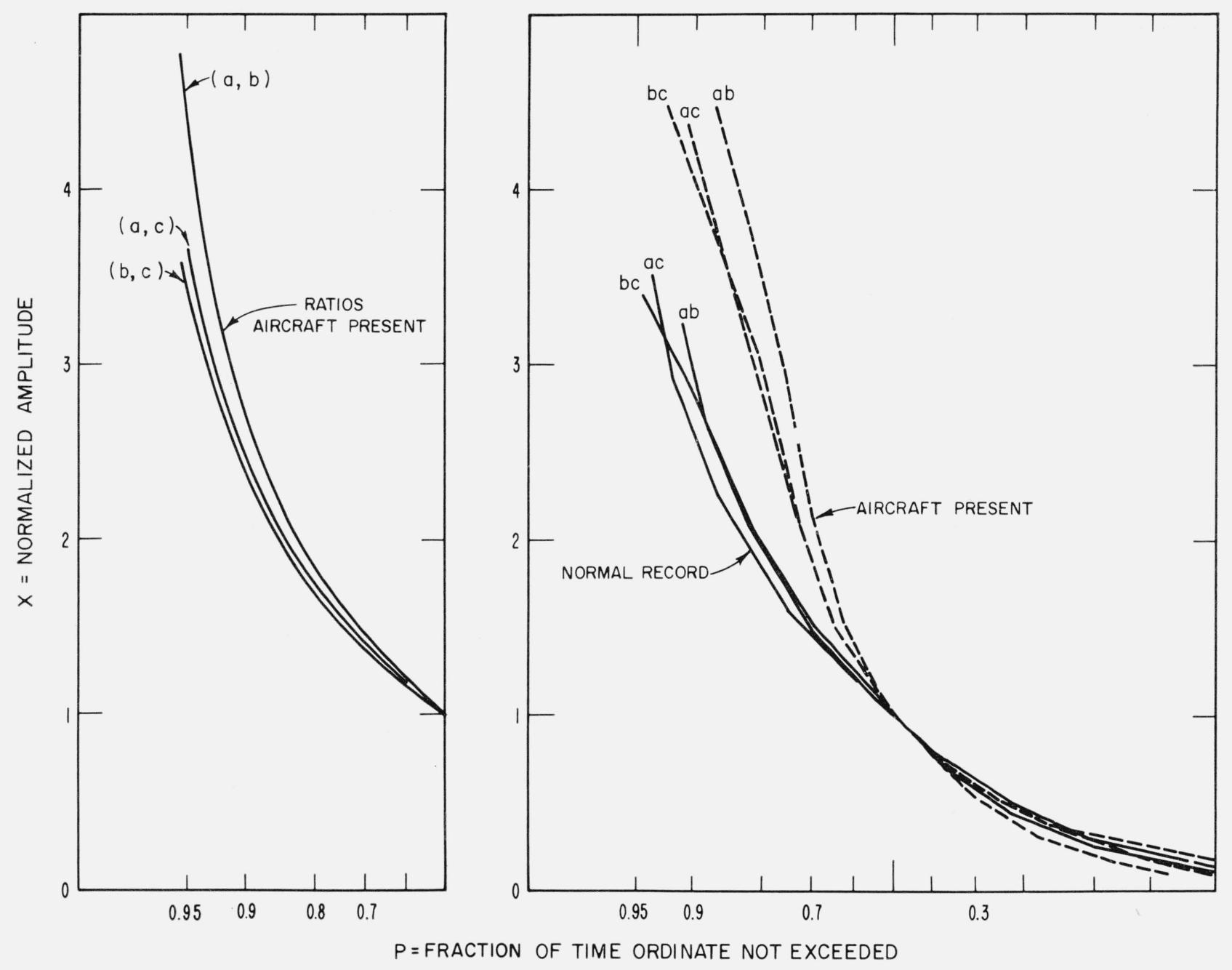

Figure 17. Ratio and product distributions from August 5, 1959.

\section{Appendix}

If two distributions derive from Gaussian populations on the complex wave vector plane, the corresponding joint distribution can be written with correlation parameters $\rho$ and $\psi$ [Wheelon, 1959],

$$
\begin{gathered}
p_{4} d \Omega=\exp \left[-\frac{1}{2}\left\{r_{1}^{2}+r_{2}^{2}-2 r_{1} r_{2} \rho \cos \left(\theta_{1}-\theta_{2}+\psi\right)\right\}\right. \\
\left.\frac{1}{1-\rho^{2}}\right] \times \frac{1}{4 \pi^{2}} \cdot \frac{r_{1} r_{2}}{1-\rho^{2}} \cdot d r_{1} d r_{2} d \theta_{1} d \theta_{2} .
\end{gathered}
$$

Here the complex wave vectors, $x_{i}+j y_{i}=r_{i} \exp j \theta_{i}(i=$ 1 or 2), are normalized so that $\left\langle x_{i}^{2}\right\rangle=\left\langle y_{i}^{2}\right\rangle=1$, and $\rho \cos \psi=\left\langle x_{1} x_{2}\right\rangle=\left\langle y_{1} y_{2}\right\rangle, \rho \sin \psi=\left\langle x_{1} y_{2}\right\rangle=$ $-\left\langle x_{2} y_{1}\right\rangle$, the brackets indicating statistical averages. Integration over $\theta_{1}$ and over $\theta_{2}$ leaves the joint distribution of two Rayleigh-distributed amplitudes:

$$
\begin{aligned}
& p_{2}\left(r_{1}, r_{2} ; \rho\right) d r_{1} d r_{2} \\
& \quad=\exp \left[-\frac{1}{2}\left(\frac{r_{1}^{2}+r_{2}^{2}}{1-\rho^{2}}\right)\right] I_{0}\left(\frac{r_{1} r_{2} \rho}{\left[1-\rho^{2}\right]}\right) \frac{r_{1} r_{2} d r_{1} d r_{2}}{1-\rho^{2}} .
\end{aligned}
$$

Now $p_{4}\left(r_{1}, r_{2}, \theta_{1}, \theta_{2} ; \rho, \psi\right)$ in (A.1) is periodic in $\left(\theta_{1}-\theta_{2}\right)$. But in our case the difference in path lengths, represented by $\theta_{1}-\theta_{2}$ for the two signals, will be distributed to peak at some value near zero, rather than to show periodicity. (A Gaussian distribution for the phase difference might be a better representation than $p_{4}$; an attempt, not wholly successful, to derive such a representation appears on page 512 of Hirai, Fukushima, and Kurihara [1960].) This objection, however, does not apply to the derived expression, $p_{2}$, which has no phase dependence; moreover, it reduces to the same Rayleigh form on integration over $r_{1}$ or $r_{2}$. It appears to provide an adequate model for much of our data on correlated Rayleigh amplitudes. 
From (A.2), after integration over the product $r_{1} r_{2}$ in the interval $(0, \infty)$ and over the ratio $r_{1} / r_{2}$ in the interval $(1, X)$, we obtain:

$\beta(\rho)=\frac{\sinh \alpha}{\left[\cosh ^{2} \alpha-\rho^{2}\right]^{1 / 2}}$, with $1+\beta$

$$
=2 P, \exp \alpha=X \text {. }
$$

From (A.2), after integration over $r_{1} / r_{2}$ in $(0, \infty)$ and over $r_{1} r_{2}$ in $(0, y)$, we obtain:

$$
\begin{aligned}
P(\bar{y}) & =\int_{0}^{\bar{y}}\left(1-\rho^{2}\right) K_{0}(y) I_{0}(\rho y) y d y \\
& =1-\bar{y}\left[I_{0}(\rho \bar{y}) K_{1}(\bar{y})+\rho K_{0}(\bar{y}) I_{1}(\rho \bar{y})\right] ; \quad y=\frac{r_{1} r_{2}}{1-\rho^{2}} .
\end{aligned}
$$

In figure 15 we plot the expressions $P(X)$ from (A.3) and (A.4) for four values of the correlation $\rho$. In plotting (A.4), the ordinate $X$ has been normalized (dividing $\bar{y}$ by its median value), so $P(1)=1 / 2$.

From (A.2) we can also determine the expected values: $\left\langle r_{1} r_{2}\right\rangle=2 E(\rho)-\left(1-\rho^{2}\right) K(\rho)$, and $\left\langle r_{1} / r_{2}\right\rangle$ $=E(\rho)$. Here $E$ and $K$ are complete elliptic integrals of the first and second kinds (while $I_{0}, I_{1}$,
$K_{n}$ and $K_{1}$ in (A.4) are Bessel functions). The expressions in question are conveniently modified to emphasize their dependence on $\rho$. We are led to define correlation parameters $R$ and $S$ thus:

$$
R=\frac{\left\langle r_{1} r_{2}\right\rangle-(\langle r\rangle)^{2}}{\left\langle r^{2}\right\rangle-(\langle r\rangle)^{2}}=0.916 \rho^{2}\left(1+\rho^{2} / 16+\ldots\right),
$$

and

$$
\begin{aligned}
S=\frac{\left\langle r_{1} \mid r_{2}\right\rangle-\langle r\rangle}{1-\langle r\rangle\langle 1 / r\rangle} & \\
& =0.688 \rho^{2}\left(1+3 \rho^{2} / 16+\ldots\right) .
\end{aligned}
$$

One expects $\rho$ to be a monotonic decreasing function of the frequency difference between signals, so $\rho(a, c)$ should be less than $\rho(a, b)$ or $\rho(b, c)$. However, there is apparently no necessary relation between these three correlations. Moreover, in a search for closed expressions for the integrals involved in $\left\langle r_{a} r_{b} r_{c}\right\rangle$, and in the cumulative distribution of the triple product, $P\left(r_{a} r_{b} r_{c}\right)$, we have had little success.

(Paper 67D4-276 